\title{
SemaKoDE: Hybrid System for Knowledge Discovery in Sensor-Based Smart Environments
}

\author{
Stefan Negru \\ Faculty of Computer Science A. I. Cuza University of Iasi, Romania \\ stefan.negru@info.uaic.ro
}

\begin{abstract}
This article describes a conceptual hybrid architecture for a knowledge discovery system, able to automatically annotate, reason, classify and operate with sensor data. The adoption of semantic web technologies to enrich sensor and link data represents an adequate methodology that facilitates the processes of reasoning, classification and other types of automation. We discussed a system deployment scenario in the context of e-health.
\end{abstract}

Keywords: Knowledge Discovery System, Sensors, Linked Data.

\section{Introduction}

The ubiquity of sensors and sensor networks brought opportunities regarding software applications in areas like smart environments, ambient management, disaster prediction and management, adaptability to climate change, security, support systems, infrastructures management etc.

Recent work has raised the idea to combine the sensor data with web technologies in order to design future services and applications. This vision is related to the aim of ubiquitous computing, as well as the proposal of "Internet of Things" 6].

We envision and design a conceptual hybrid knowledge discovery system, able to automatically annotate, reason, classify and operate with sensor data. Although several similar sensor network systems exist [8]5], our system proposal also takes into consideration additional factors like evaluation and re-usability of the discovered knowledge in different contexts, thus making it available to other services and applications.

\section{System Architecture}

The SemaKoDE (Semantic Knowledge Discovery Environment) system consists of two main components: the first one is similar to a classical KDD system [7, and provides an established and well researched base for identifying patterns or models in data. The second component takes advantage of semantic 
web technologies which facilitate the reusing and sharing of information, within the system and with other systems. Our system is divided into the following layers: Knowledge Base Layer, Network Management Layer, Database Layer, Discovery Layer, Application Layer (Fig. 1).

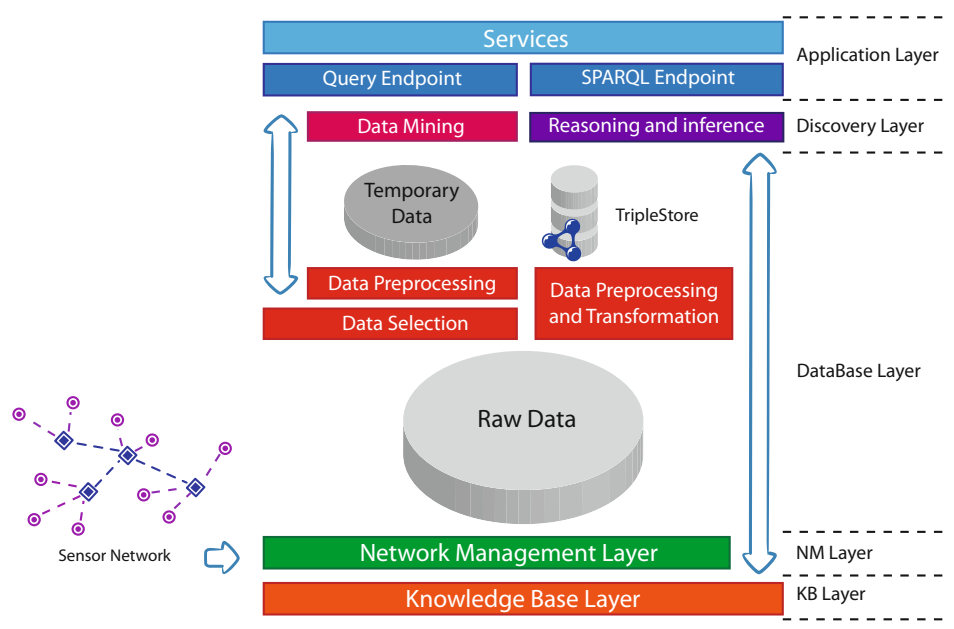

Fig. 1. SemaKoDE System Architecture

Knowledge Base Layer. This layer contains the axioms defining the classes and relations in the ontology along with the logic operations which are used to perform inferences - the TBox 14 .

Network Management Layer. The main responsibility of the Network Management Layer is to facilitate collecting data for the Database Layer and also to manage the functions related to the interaction between multiple sensors. Other aspects, such as network hierarchy and clustering of sensor, nodes are also done at this layer.

Database Layer. Data Selection and Data Preprocessing sub-layers correspond to two of the steps from the KDD process [2. The Data Preprocessing and Transformation sub-layer handles similar functions but, in addition, offers support for semantically annotating sensor data and storing this data into a triple store [1] (ABox [14]).

Discovery Layer. The Discovery Layer encapsulates both data mining [7] and reasoning algorithms as presented in [14, used for knowledge discovery. Although the types of algorithms have roughly the same purpose, we believe that patterns/models obtained via data mining and the inferred information via reasoning provide slightly different results. Thereby, we want to integrate them into the Application layer in order to improve the discovery process accuracy and the knowledge quality. Regarding evaluating the knowledge quality, we take 
into consideration methods for evaluating triple-stores [9], or the ontology quality [10].

Application Layer. The top level layer handles the data interpretation (extracting knowledge) and data integration too. As the KDD process operates with user predefined goals, this layer also provides a user interface which handles the user interaction, as we need to represent the discovered knowledge in an easy to understand manner.

Considering the query endpoints, two methods are to be explored. One in which we keep the current structure (Fig.1) with the two query endpoints (classical query service and SPARQL endpoint), the integration being done via several services, and one in which the two query endpoints are unified into a single service, using a future SPARQL extension. Next versions of the SemaKoDE system will incorporate predictive capabilities, via extensions implemented at the Application Layer.

\section{System Deployment Scenario}

In order to illustrate SemaKoDE usefulness and versatility, we imagine a scenario centered around hospital building environment. The hospital maintains a patient database (that stores diseases, disease symptoms, disabilities, patient rooms etc). The hospital building has several spatially distributed sensors, that monitor the environment. SemaKoDE system will act as a bridge linking the data between the database and sensor network in order to extend the overall knowledge about this environment. For example, certain environmental factors (detected via the sensor network) might cause the patient health status to aggravate, depending on the disease (Disease Ontology - http://www.disease-ontology.org/).

In case of major event (hazard) such as a fire, the system will make use of its knowledge discovery capabilities. First it confirms the fire is real, by linking data

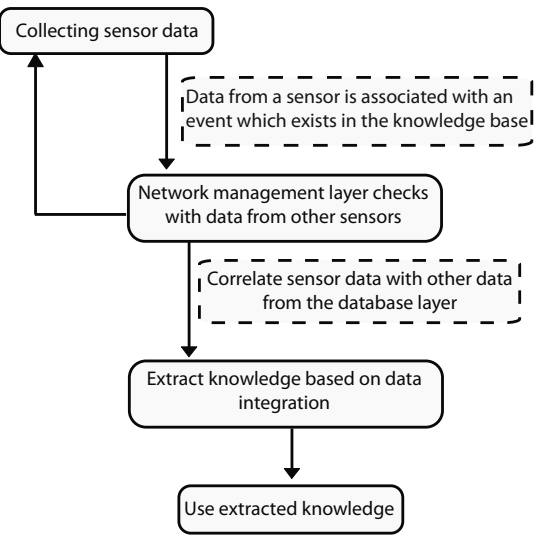

Fig. 2. SemaKoDE Main Processes 
from various sensors from the sensor network and deploying the fire sprinklers. Operations are done by the Network Management layer as represented in Fig. 2 . After confirming the fire, the system proceeds to provide a list of patients located near the fire, having a high risk factor (e.g. patients that have certain disabilities or have trouble breathing and can not evacuate themselves ). The predictive capabilities of the system come in handy in providing a fire spreading pattern.

\section{Conclusions and Future Work}

This paper presented SemaKoDE, a hybrid system used for knowledge discovery in sensor-based smart environments. Our system proposal combines traditional knowledge discovery, based on data mining, with ontology based knowledge discovery, in order to create not only a well optimized KDS, but also a system able to share and reuse data. Our further direction of research is focused on developing, testing and improving such a system, in order to constantly perfect the way it can collect, process and operate with sensor data, with the purpose of using it in real world scenarios, similar with those presented in this paper.

Acknowledgements. This work was supported by the European Social Fund in Romania, under the responsibility of the Managing Authority for the Sectorial Operational Program for Human Resources Development 2007-2013 [grant POSDRU/107/1.5/S/78342].

\section{References}

1. Baader, F., et al.: The Description Logic Handbook. Cambridge University Press (2007)

2. Fayyad, U., et al.: From data mining to knowledge discovery: an overview. AI Magazine, 37-54 (1996)

3. Guo, Y., Pan, Z., Heflin, J.: An Evaluation of Knowledge Base Systems for Large OWL Datasets. In: McIlraith, S.A., Plexousakis, D., van Harmelen, F. (eds.) ISWC 2004. LNCS, vol. 3298, pp. 274-288. Springer, Heidelberg (2004)

4. Harmelen, F.V., et al.: Handbook of knowledge representation. Elsevier (2008)

5. Huang, V., Javed, M.K.: Semantic sensor information description and processing. In: 2nd International Conference on Sensor Technologies and Applications, pp. 456-461. IEEE (2008)

6. International Telecommunication Union: ITU Internet Report 2005: The Internet of Things (2005)

7. Leondes, C.T.: Knowledge-based systems: techniques and applications. Academic Press (2000)

8. Moraru, A., et al.: Using semantic annotation for knowledge extraction from geographically distributed and heterogeneous sensor data. In: 4th SensorKDD. ACM (2010)

9. Rohloff, K., Dean, M., Emmons, I., Ryder, D., Sumner, J.: An Evaluation of TripleStore Technologies for Large Data Stores. In: Meersman, R., Tari, Z. (eds.) OTMWS 2007, Part II. LNCS, vol. 4806, pp. 1105-1114. Springer, Heidelberg (2007)

10. Stvilia, B.: A model for ontology quality evaluation. First Monday (2007)

11. Yeh, C., Lin, R.: Design and Implementation of an RDF Triple Store. In: Proceedings of the 1st Workshop of DATF. Academia Sinica (2002) 